\title{
Polynomiality of certain average weights for oscillating tableaux
}

\author{
Guo-Niu Han Huan Xiong* \\ Institut de Recherche Mathématique Avancée \\ Université de Strasbourg \\ F-67000 Strasbourg, France \\ \{guoniu, xiong\}@math.unistra.fr
}

Submitted: Mar 12, 2018; Accepted: Aug 20, 2018; Published: Oct 5, 2018

(C) The authors. Released under the CC BY-ND license (International 4.0).

\begin{abstract}
We prove that a family of average weights for oscillating tableaux are polynomials in two variables, namely, the length of the oscillating tableau and the size of the ending partition, which generalizes a result of Hopkins and Zhang. Several explicit and asymptotic formulas for the average weights are also derived. The main idea in this paper is to translate the study of certain average weights for oscillating tableaux to the study of an operator $\Psi$ from the set of real coefficient polynomials with two parameters to itself.
\end{abstract}

Mathematics Subject Classifications: 05A15, 05A17, 05A19, 11P81

\section{Introduction}

Background on partitions and Young's lattice can be found in $[19,27]$. A partition is a finite weakly decreasing sequence of positive integers $\lambda=\left(a_{1}, a_{2}, \ldots, a_{L}\right)$, where $a_{i}(1 \leqslant$ $i \leqslant L)$ are called the parts of $\lambda$. The integer $|\lambda|=\sum_{1 \leqslant i \leqslant L} a_{i}$ is called the size of $\lambda$. Let $\mathbb{P}$ be the set of all partitions. The partition $\lambda$ is identified with its Young diagram, which is a collection of boxes arranged in left-justified rows with $a_{i}$ boxes in the $i$-th row. A standard Young tableau of shape $\lambda$ is a filling in the boxes of the Young diagram of $\lambda$ with distinct numbers from 1 to $|\lambda|$ such that the numbers in each row and each column are increasing (see Figure 1). Equivalently, a standard Young tableau of shape $\lambda$ can be seen as a sequence of partitions $T=\left(\lambda^{0}, \lambda^{1}, \ldots, \lambda^{l}\right)$ such that $\lambda^{0}=\emptyset, \lambda^{l}=\lambda$; and $\lambda^{i+1}$ is obtained by adding a box to $\lambda^{i}$ for $0 \leqslant i \leqslant l-1$. Denote by $f_{\lambda}$ the number of

\footnotetext{
${ }^{*}$ Corresponding author. Supported by Grant [P2ZHP2_171879] of the Swiss National Science Foundation.
} 
standard Young tableaux of shape $\lambda$. In 1954, Frame, Robinson and Thrall [8] proved the following celebrated hook length formula, which shows that the number of standard Young tableaux of shape $\lambda$ is determined by hook lengths of $\lambda$ :

$$
f_{\lambda}=\frac{|\lambda| !}{H_{\lambda}}
$$

where $H_{\lambda}$ is the product of all hook lengths of boxes in the Young diagram of $\lambda$. Various proofs of the above hook length formula were given in $[1,10,16,20]$.
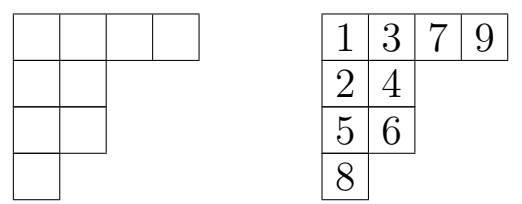

Figure 1: The Young diagram of the partition $(4,2,2,1)$ and a standard Young tableau of shape $(4,2,2,1)$.

Oscillating tableaux are generalizations of standard Young tableaux. An oscillating tableau of shape $\lambda$ and length $l$ is a sequence of partitions $T=\left(\lambda^{0}, \lambda^{1}, \ldots, \lambda^{l}\right)$ such that $\lambda^{0}=\emptyset, \lambda^{l}=\lambda$; and $\left|\lambda^{i} / \lambda^{i+1}\right|=1$ or $\left|\lambda^{i+1} / \lambda^{i}\right|=1$ for each $0 \leqslant i \leqslant l-1$, i.e., $\lambda^{i+1}$ is obtained by adding a box to or removing a box from $\lambda^{i}$ (see [14, 21]). Therefore the oscillating tableau can be seen as a walk from $\emptyset$ to $\lambda$ in Young's lattice (see [28] and Figure 2).

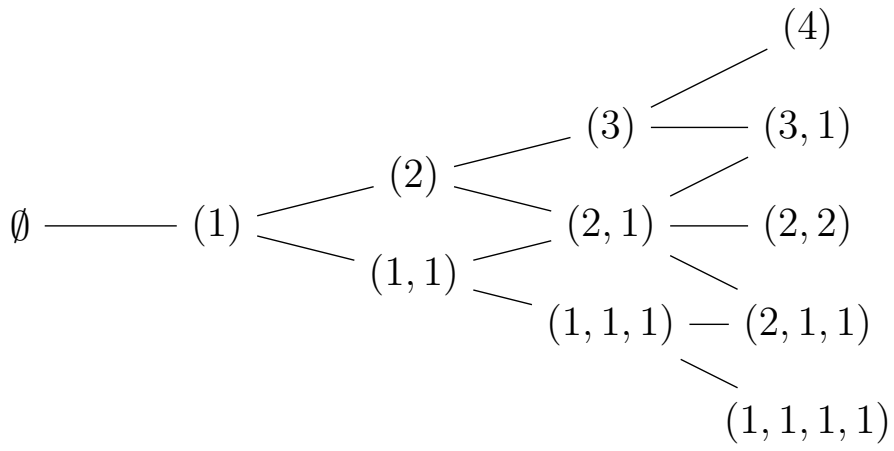

Figure 2: The Young's lattice of partitions with sizes at most 4.

Let $\mathcal{O T}(\lambda, l)$ be the set of oscillating tableaux of shape $\lambda$ and length $l$. The cardinality of $\mathcal{O T}(\lambda, l)$ is well-known to be the following.

Theorem 1 ([24, 26, 28, 29]). Let $\lambda \in \mathbb{P}$ and $k=|\lambda|$. Then for all $n \in \mathbb{N}$, we have

$$
\# \mathcal{O} \mathcal{T}(\lambda, k+2 n)=\left(\begin{array}{c}
k+2 n \\
k
\end{array}\right)(2 n-1) ! ! f_{\lambda} .
$$

On the other hand, $\# \mathcal{O T}(\lambda, l)=0$ if $l \neq k+2 n$ for any $n \in \mathbb{N}$. 
Bijective proofs of Theorem 1 were given in [24, 29]. Another proof was obtained by Stanley $[26,28]$ in the study of differential posets. The enumerations of various oscillating tableaux with restrictive conditions can be found in [3, 4, 6, 17, 18, 23]. In 2015, Hopkins and Zhang [14] proved the following result on the average of a certain weight function of oscillating tableaux. Their proof is motivated by Stanley's theory of differential posets $[26,28]$.

Theorem 2 ([14]). Let $\lambda \in \mathbb{P}$ and $k=|\lambda|$. Then for all $n \in \mathbb{N}$,

$$
\frac{1}{\# \mathcal{O} \mathcal{T}(\lambda, k+2 n)} \sum_{T \in \mathcal{O} \mathcal{T}(\lambda, k+2 n)} \operatorname{wt}(T)=(k+2 n+1) \cdot \frac{3 k+2 n}{6},
$$

where $\operatorname{wt}(T):=\sum_{i=0}^{l}\left|\lambda^{i}\right|$ for each oscillating tableau $T=\left(\lambda^{0}, \ldots, \lambda^{l}\right)$.

As noted by Hopkins and Zhang [14], it is surprising that the above average in Theorem 2 is a polynomial of $n$ and $|\lambda|$. In this paper, we generalize Theorem 2 and show that this polynomiality holds for a family of weight functions for oscillating tableaux. The following is our main result.

Theorem 3. Let $P(x, y)$ be a given polynomial of two variables $x$ and $y$. For each oscillating tableau $T=\left(\lambda^{0}, \ldots, \lambda^{l}\right)$, let $\operatorname{wt}_{P}(T):=\sum_{i=0}^{l} P\left(\left|\lambda^{i}\right|, i\right)$. Then there exists a polynomial $Q(x, y)$ with the same degree and constant term as $P(x, y)$ such that

$$
\frac{1}{\# \mathcal{O} \mathcal{T}(\lambda,|\lambda|+2 n)} \sum_{T \in \mathcal{O} \mathcal{T}(\lambda,|\lambda|+2 n)} \operatorname{wt}_{P}(T)=(|\lambda|+2 n+1) Q(|\lambda|,|\lambda|+2 n)
$$

for any $n \in \mathbb{N}$ and $\lambda \in \mathbb{P}$.

Theorem 3 tells us that the average of the weight function $\operatorname{wt}_{P}(T)$ is a polynomial of $n$ and $|\lambda|$ with degree $\operatorname{deg}(P)+1$. In fact, the polynomial $Q(x, y)$ in Theorem 3 equals $\Psi^{-1}(P(x, x+2 y))$, where $\Psi$ is an operator given in Definition 10. Usually, it is not easy to compute the explicit formula for $Q(x, y)$ when $P(x, y)$ is given except for some special cases that we will describe below. For example, letting $\lambda=\emptyset$ and $P(x, y)=\left(\begin{array}{l}x \\ r\end{array}\right)$ where $r \in \mathbb{N}$, we can derive the following explicit formulas.

Corollary 4. For any $n, r \in \mathbb{N}$, we have

$$
\frac{2^{n} n !}{(2 n+1) !} \quad \sum_{\left(\lambda^{0}, \lambda^{1}, \ldots, \lambda^{2 n}\right) \in \mathcal{O} \mathcal{T}(\emptyset, 2 n)} \sum_{i=0}^{2 n}\left(\begin{array}{c}
\left|\lambda^{i}\right| \\
r
\end{array}\right)=\frac{2^{r} r !^{2}}{(2 r+1) !}\left(\begin{array}{l}
n \\
r
\end{array}\right) .
$$

Also, by letting $P(x, y)=x$ in Theorem 3, we derive Theorem 2. For each oscillating tableau $T=\left(\lambda^{0}, \ldots, \lambda^{l}\right)$, let

$$
\mathrm{wt}_{a, b}(T):=\sum_{i=0}^{l}\left|\lambda^{i}\right|^{a} \cdot i^{b}
$$

where $a, b \in \mathbb{N}$. Then, we obtain the following corollaries for the weight functions $P(x, y)=x^{2}$ and $x y$ respectively. 
Corollary 5. Let $\lambda \in \mathbb{P}$ and $k=|\lambda|$. Then for any $n \in \mathbb{N}$, we have

$$
\frac{1}{\# \mathcal{O} \mathcal{T}(\lambda, k+2 n)} \sum_{T \in \mathcal{O} \mathcal{T}(\lambda, k+2 n)} \mathrm{wt}_{2,0}(T)=(k+2 n+1) \cdot \frac{10 k^{2}+4 n^{2}+10 k n+5 k+6 n}{30} .
$$

Corollary 6. Let $\lambda \in \mathbb{P}$ and $k=|\lambda|$. Then for any $n \in \mathbb{N}$,

$$
\frac{1}{\# \mathcal{O} \mathcal{T}(\lambda, k+2 n)} \sum_{T \in \mathcal{O} \mathcal{T}(\lambda, k+2 n)} \mathrm{wt}_{1,1}(T)=(k+2 n+1) \cdot \frac{2 k^{2}+2 n^{2}+5 k n+k}{6} .
$$

For the general weight function $\mathrm{wt}_{i, j}(T)$, we can derive the following asymptotic formulas of their averages.

Theorem 7. Let $i, j \in \mathbb{N}$. For a fixed partition $\lambda$, we have

$$
\frac{1}{\# \mathcal{O} \mathcal{T}(\lambda,|\lambda|+2 n)} \sum_{T \in \mathcal{O} \mathcal{T}(\lambda,|\lambda|+2 n)} \mathrm{wt}_{i, j}(T)=\frac{i !(i+j) !(2 n)^{i+j+1}}{(2 i+j+1) !}+O\left(n^{i+j}\right)
$$

when $n \rightarrow \infty$.

For a fixed nonnegative integer $n$, we have

$$
\frac{1}{\# \mathcal{O} \mathcal{T}(\lambda,|\lambda|+2 n)} \sum_{T \in \mathcal{O} \mathcal{T}(\lambda,|\lambda|+2 n)} \mathrm{wt}_{i, j}(T)=\frac{|\lambda|^{i+j+1}}{i+j+1}+O\left(|\lambda|^{i+j}\right)
$$

when $|\lambda| \rightarrow \infty$.

The main idea in the proof of Theorem 3 is to translate the study of certain average weights for oscillating tableaux to the study of the operator $\Psi$ given in Definition 10, which is shown to be a bijection from the set of real coefficient polynomials with two parameters to itself (see Theorem 11).

\section{Proofs of main results}

In this section, we prove Theorem 3 and the three corollaries stated in the introduction. Let $\lambda$ be a partition. Denote by $\Omega^{+}(\lambda)$ (resp. $\Omega^{-}(\lambda)$ ) the set of partitions $\lambda^{+}$(resp. $\lambda^{-}$) obtained by adding (resp. removing) a box to (resp. from) $\lambda$ :

$$
\Omega^{+}(\lambda):=\left\{\lambda^{+}:\left|\lambda^{+} / \lambda\right|=1\right\}
$$

and

$$
\Omega^{-}(\lambda):=\left\{\lambda^{-}:\left|\lambda / \lambda^{-}\right|=1\right\} .
$$

Example 8. For the partition $\lambda=(5,2,2,1)$, we have

$$
\Omega^{+}(\lambda):=\{(6,2,2,1),(5,3,2,1),(5,2,2,2),(5,2,2,1,1)\}
$$

and

$$
\Omega^{-}(\lambda):=\{(4,2,2,1),(5,2,1,1),(5,2,2)\} .
$$


We need the following lemma.

Lemma 9. Let $\lambda \in \mathbb{P}$ and $k=|\lambda|$. For any $n \in \mathbb{N}$ with $k+2 n>0$ we have

$$
\frac{\sum_{\lambda^{+} \in \Omega^{+}(\lambda)} \# \mathcal{O} \mathcal{T}\left(\lambda^{+}, k+2 n-1\right)}{\# \mathcal{O} \mathcal{T}(\lambda, k+2 n)}=\frac{2 n}{k+2 n}
$$

and

$$
\frac{\sum_{\lambda^{-} \in \Omega^{-}(\lambda)} \# \mathcal{O T}\left(\lambda^{-}, k+2 n-1\right)}{\# \mathcal{O} \mathcal{T}(\lambda, k+2 n)}=\frac{k}{k+2 n} .
$$

Proof. For $\lambda^{+} \in \Omega^{+}(\lambda)$ and $\lambda^{-} \in \Omega^{-}(\lambda)$, by Theorem 1, we obtain

$$
\frac{\# \mathcal{O} \mathcal{T}\left(\lambda^{+}, k+2 n-1\right)}{\# \mathcal{O} \mathcal{T}(\lambda, k+2 n)}=\frac{2 n}{k+2 n} \frac{f_{\lambda^{+}}}{(k+1) f_{\lambda}}
$$

and

$$
\frac{\# \mathcal{O} \mathcal{T}\left(\lambda^{-}, k+2 n-1\right)}{\# \mathcal{O} \mathcal{T}(\lambda, k+2 n)}=\frac{k}{k+2 n} \frac{f_{\lambda^{-}}}{f_{\lambda}}
$$

But it is well known that $\sum_{\lambda^{+} \in \Omega^{+}(\lambda)} f_{\lambda^{+}}=(k+1) f_{\lambda}$ and $\sum_{\lambda^{-} \in \Omega^{-}(\lambda)} f_{\lambda^{-}}=f_{\lambda}$ (for example, see [11, Lemmas 2.2 and 2.3]). Then the proof is complete.

Let $\mathbb{R}[x, y]$ be the set of polynomials of $x$ and $y$ with real coefficients. For each nonnegative integer $r$, let $\mathbb{R}_{r}[x, y]$ be the set of polynomials in $\mathbb{R}[x, y]$ with degrees at most $r$.

Definition 10. The operator $\Psi: \mathbb{R}[x, y] \rightarrow \mathbb{R}[x, y]$ is defined by

$\Psi(A(x, y)):=(x+2 y+1) A(x, x+2 y)-x A(x-1, x+2 y-1)-2 y A(x+1, x+2 y-1)$

for each polynomial $A(x, y) \in \mathbb{R}[x, y]$.

Theorem 11. Let $r \in \mathbb{N}$. The operator $\Psi$ provides a bijection between $\mathbb{R}_{r}[x, y]$ and itself. Furthermore, $A(x, y)$ and $\Psi(A(x, y))$ have the same degree and constant term for each polynomial $A(x, y) \in \mathbb{R}[x, y]$.

Proof. It is obvious that the operator $\Psi$ is an $\mathbb{R}$-linear map over $\mathbb{R}[x, y]$. For $0 \leqslant i \leqslant r$ we have

$$
\begin{aligned}
\Psi\left(x^{i} y^{r-i}\right)= & (x+2 y+1) x^{i}(x+2 y)^{r-i}-x(x-1)^{i}(x+2 y-1)^{r-i} \\
& \quad-2 y(x+1)^{i}(x+2 y-1)^{r-i} \\
= & (r+i+1) x^{i}(x+2 y)^{r-i}-i x^{i-1}(x+2 y)^{r-i+1}+K_{r, i}(x, y)
\end{aligned}
$$

for some polynomial $K_{r, i}(x, y)$ with degree at most $r-1$. Let $\alpha_{j, i}(x, y):=x^{i} y^{j-i}$ and $\beta_{j, i}(x, y)=x^{i}(x+2 y)^{j-i}$ for $0 \leqslant i \leqslant j$. Then $\left\{\alpha_{j, i}: 0 \leqslant i \leqslant j \leqslant r\right\}$ and $\left\{\beta_{j, i}: 0 \leqslant i \leqslant\right.$ $j \leqslant r\}$ form two bases for the $\mathbb{R}$-linear space $\mathbb{R}_{r}[x, y]$. From (6) we have

$$
\Psi\left(\alpha_{r, r}, \alpha_{r, r-1}, \ldots, \alpha_{r, 0}, \alpha_{r-1, r-1}, \alpha_{r-1, r-2}, \ldots, \alpha_{r-1,0}, \ldots, \alpha_{1,1}, \alpha_{1,0}, \alpha_{0,0}\right)
$$




$$
=\left(\beta_{r, r}, \beta_{r, r-1}, \ldots, \beta_{r, 0}, \beta_{r-1, r-1}, \beta_{r-1, r-2}, \ldots, \beta_{r-1,0}, \ldots, \beta_{1,1}, \beta_{1,0}, \beta_{0,0}\right) \times M_{r}
$$

where

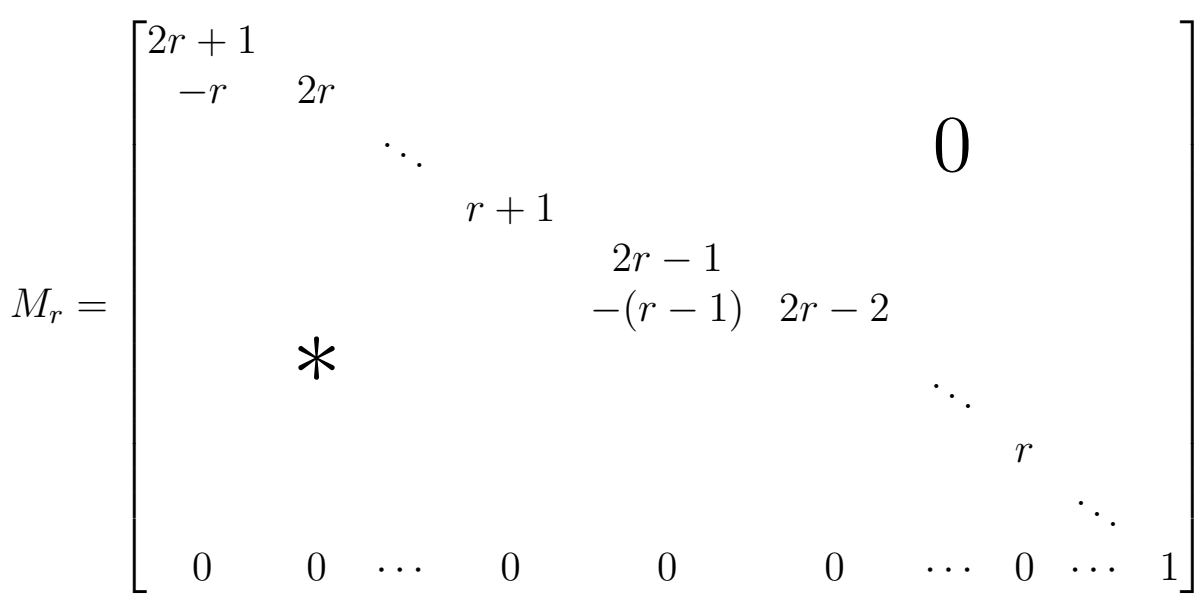

is an invertible lower triangular matrix with diagonal entries $\{2 r+1,2 r, \ldots, r+1 ; 2 r-$ $1,2 r-2, \ldots, r ; \ldots ; 9,8,7,6,5 ; 7,6,5,4 ; 5,4,3 ; 3,2 ; 1\}$. For example, we have

$$
M_{2}=\left[\begin{array}{cccccc}
5 & 0 & 0 & 0 & 0 & 0 \\
-2 & 4 & 0 & 0 & 0 & 0 \\
0 & -1 & 3 & 0 & 0 & 0 \\
0 & -2 & 0 & 3 & 0 & 0 \\
-1 & 1 & -1 & -1 & 2 & 0 \\
0 & 0 & 0 & 0 & 0 & 1
\end{array}\right]
$$

Then $\Psi$ must be an isomorphism from the $\mathbb{R}$-linear space $\mathbb{R}_{r}[x, y]$ to itself. It is obvious that $A(x, y)$ and $\Psi(A(x, y))$ have the same degree and constant term.

Now we are ready to give the proof of Theorem 3 .

Proof of Theorem 3. Let $k=|\lambda|$ and $Q(x, y)=\Psi^{-1}(P(x, x+2 y))$. Actually we will prove the following identity

$$
\sum_{T \in \mathcal{O} \mathcal{T}(\lambda, k+2 n)} \operatorname{wt}_{P}(T)=\# \mathcal{O} \mathcal{T}(\lambda, k+2 n) \times(k+2 n+1) Q(k, k+2 n)
$$

for any $n \in \mathbb{Z}$ and $\lambda \in \mathbb{P}$ with $k+2 n \geqslant 0$ by induction on $k+2 n$. For each oscillating tableaux

$$
T=\left(\lambda^{0}, \ldots, \lambda^{k+2 n}\right) \in \mathcal{O} \mathcal{T}(\lambda, k+2 n),
$$

we have $\lambda^{k+2 n-1} \in \Omega^{+}(\lambda) \cup \Omega^{-}(\lambda)$. Therefore

$$
\begin{aligned}
\sum_{T \in \mathcal{O} \mathcal{T}(\lambda, k+2 n)} \operatorname{wt}_{P}(T)= & \sum_{\lambda^{+} \in \Omega^{+}(\lambda)} \sum_{T^{+} \in \mathcal{O} \mathcal{T}\left(\lambda^{+}, k+2 n-1\right)}\left(\operatorname{wt}_{P}\left(T^{+}\right)+P(k, k+2 n)\right) \\
& +\sum_{\lambda^{-} \in \Omega^{-}(\lambda)} \sum_{T^{-} \in \mathcal{O} \mathcal{T}\left(\lambda^{-}, k+2 n-1\right)}\left(\mathrm{wt}_{P}\left(T^{-}\right)+P(k, k+2 n)\right)
\end{aligned}
$$




$$
\begin{aligned}
& =\sum_{\substack{\lambda^{+} \in \Omega^{+}(\lambda)\\
}} \sum_{\substack{T^{+} \in \mathcal{O} \mathcal{T}\left(\lambda^{+}, k+2 n-1\right) \\
\lambda^{-} \in \Omega^{-}(\lambda)}} \sum_{\substack{T^{-} \in \mathcal{O} \mathcal{T}\left(\lambda^{-}, k+2 n-1\right) \\
\operatorname{wt}_{P}}} \operatorname{wt}_{P}\left(T^{-}\right) \\
& +P(k, k+2 n) \times \# \mathcal{O T}(\lambda, k+2 n) .
\end{aligned}
$$

Let

$$
A(x, y):=(x+2 y+1) Q(x, x+2 y) .
$$

When $k+2 n=0$, it is obvious that

$$
\sum_{T \in \mathcal{O} \mathcal{T}(\lambda, 0)} \operatorname{wt}_{P}(T)=\# \mathcal{O} \mathcal{T}(\lambda, 0) \times A(k, n)
$$

for any $\lambda \in \mathbb{P}$ by Theorem 11 .

When $k+2 n \geqslant 1$, by the induction hypothesis, identity (9) becomes

$$
\begin{aligned}
\sum_{T \in \mathcal{O} \mathcal{T}(\lambda, k+2 n)} \operatorname{wt}_{P}(T)= & \sum_{\lambda^{+} \in \Omega^{+}(\lambda)} A(k+1, n-1) \# \mathcal{O} \mathcal{T}\left(\lambda^{+}, k+2 n-1\right) \\
& +\sum_{\lambda^{-} \in \Omega^{-}(\lambda)} A(k-1, n) \# \mathcal{O} \mathcal{T}\left(\lambda^{-}, k+2 n-1\right) \\
& +P(k, k+2 n) \# \mathcal{O} \mathcal{T}(\lambda, k+2 n) .
\end{aligned}
$$

When $n<0$, the above summation equals 0 and thus (8) is true. When $n \geqslant 0$, by Lemma 9 , the above summation is equal to

$$
\# \mathcal{O} \mathcal{T}(\lambda, k+2 n)\left(\frac{2 n}{k+2 n} A(k+1, n-1)+\frac{k}{k+2 n} A(k-1, n)+P(k, k+2 n)\right) .
$$

By the definition of $\Psi$, we have

$$
\begin{aligned}
& P(x, x+2 y)=\Psi(Q(x, y)) \\
= & (x+2 y+1) Q(x, x+2 y)-x Q(x-1, x+2 y-1)-2 y Q(x+1, x+2 y-1) \\
= & A(x, y)-\frac{x}{x+2 y} A(x-1, y)-\frac{2 y}{x+2 y} A(x+1, y-1) .
\end{aligned}
$$

Hence, $(11)$ equals $\# \mathcal{O} \mathcal{T}(\lambda, k+2 n) \times A(k, n)$, which completes the proof.

By Theorem 3, to evaluate the average of the weight function $P(x, y)$ for oscillating tableaux, we only need to calculate $\Psi^{-1}(P(x, x+2 y))$. However it seems that the inverse of $\Psi$ has no explicit formula for general polynomial $P(x, y)$, except for some special cases.

Example 12. Let $P(x, y)=\left(\begin{array}{l}y \\ r\end{array}\right)$ where $r \in \mathbb{N}$. By Definition 10 we obtain

$$
\Psi\left(\left(\begin{array}{l}
y \\
r
\end{array}\right)\right)=(x+2 y+1)\left(\begin{array}{c}
x+2 y \\
r
\end{array}\right)-x\left(\begin{array}{c}
x+2 y-1 \\
r
\end{array}\right)-2 y\left(\begin{array}{c}
x+2 y-1 \\
r
\end{array}\right)
$$




$$
=(r+1)\left(\begin{array}{c}
x+2 y \\
r
\end{array}\right)
$$

Hence,

$$
Q(x, y)=\Psi^{-1}(P(x, x+2 y))=\Psi^{-1}\left(\left(\begin{array}{c}
x+2 y \\
r
\end{array}\right)\right)=\frac{1}{r+1}\left(\begin{array}{c}
y \\
r
\end{array}\right) .
$$

Therefore by (2), for any $n, r \in \mathbb{N}$ we have

$$
\frac{1}{\# \mathcal{O} \mathcal{T}(\lambda,|\lambda|+2 n)} \sum_{T \in \mathcal{O} \mathcal{T}(\lambda,|\lambda|+2 n)} \sum_{i=0}^{|\lambda|+2 n}\left(\begin{array}{l}
i \\
r
\end{array}\right)=(|\lambda|+2 n+1) \times \frac{1}{r+1}\left(\begin{array}{c}
|\lambda|+2 n \\
r
\end{array}\right) .
$$

The above identity can also be derived by direct calculation with the help of

$$
\sum_{i=0}^{m}\left(\begin{array}{l}
i \\
r
\end{array}\right)=\left(\begin{array}{c}
m+1 \\
r+1
\end{array}\right)=\frac{m+1}{r+1}\left(\begin{array}{c}
m \\
r
\end{array}\right)
$$

Next we give the proofs for Corollaries 4, 5 and 6 .

Proof of Corollary 4. Let $\lambda=\emptyset$ and $P(x, y)=\left(\begin{array}{l}x \\ r\end{array}\right)$ in Theorem 3. By Lemma 11 and $\mathcal{O T}(\emptyset, 2 n)=(2 n-1) !$ ! we obtain that

$$
\frac{2^{n} n !}{(2 n+1) !} \sum_{\left(\lambda^{0}, \lambda^{1}, \ldots, \lambda^{2 n}\right) \in \mathcal{O} \mathcal{T}(\emptyset, 2 n)} \sum_{i=0}^{2 n}\left(\begin{array}{c}
\left|\lambda^{i}\right| \\
r
\end{array}\right)
$$

is a polynomial $B(n)$ of $n$ with degree at most $r$. It is obvious that $B(n)=0$ for $0 \leqslant n \leqslant r-1$ and

$$
B(r)=\frac{2^{r} r !}{(2 r+1) !} \sum_{|\lambda|=r} f_{\lambda}^{2}\left(\begin{array}{l}
r \\
r
\end{array}\right)=\frac{2^{r} r !^{2}}{(2 r+1) !} .
$$

The last equality is due to the well-known formula (see $[19,27]) \sum_{|\lambda|=r} f_{\lambda}^{2}=r$ !. Since $\operatorname{deg}(B(n)) \leqslant r$, we obtain that $B(n)=\frac{2^{r} r !^{2}}{(2 r+1) !}\left(\begin{array}{l}n \\ r\end{array}\right)$. The proof is complete.

Proofs of Corollaries 5 and 6 . First we have

$$
\begin{aligned}
\Psi(x) & =2 x-2 y, \\
\Psi(y) & =2 x+4 y \\
\Psi(x y) & =3 x^{2}+4 x y-4 y^{2}-x+2 y, \\
\Psi\left(x^{2}\right) & =3 x^{2}-4 x y-x-2 y, \\
\Psi\left(y^{2}\right) & =3 x^{2}+12 x y+12 y^{2}-x-2 y .
\end{aligned}
$$

Then by linearity,

$$
\Psi\left(\frac{6 x^{2}+3 x y+y^{2}+2 x+3 y}{30}\right)=x^{2} .
$$


In Theorem 3 , let $P(x, y)=x^{2}$. Then

$$
Q(x, y)=\Psi^{-1}(P(x, x+2 y))=\Psi^{-1}\left(x^{2}\right)=\frac{6 x^{2}+3 x y+y^{2}+2 x+3 y}{30} .
$$

Therefore Corollary 5 holds by (2). Also we have

$$
\Psi\left(\frac{3 x y+y^{2}+2 x}{12}\right)=x(x+2 y) .
$$

In Theorem 3 , let $P(x, y)=x y$. Then

$$
Q(x, y)=\Psi^{-1}(P(x, x+2 y))=\Psi^{-1}(x(x+2 y))=\frac{3 x y+y^{2}+2 x}{12} .
$$

By (2) we obtain Corollary 6.

\section{Proofs of the asymptotic formulas}

In this section, we will prove Theorem 7 .

Proof of Theorem $\%$. Let $I=\{(r, i) \in \mathbb{N} \times \mathbb{N}: i \leqslant r\}$. For two elements in $I$, we say that $\left(r^{\prime}, i^{\prime}\right) \preceq(r, i)$ if $(1) r^{\prime}<r$ or $(2) r^{\prime}=r$ and $i^{\prime} \leqslant i$. It is easy to check that ' $\preceq$ ' is a partial order relation on the set $I$. Let $M_{r}, \alpha_{j, i}$ and $\beta_{j, i}$ be the same as in the proof of Theorem 11. For $0 \leqslant i \leqslant r$, by (7) we obtain

$$
\begin{aligned}
& \left(\beta_{r, r}, \beta_{r, r-1}, \ldots, \beta_{r, 0}, \beta_{r-1, r-1}, \beta_{r-1, r-2}, \ldots, \beta_{r-1,0}, \ldots, \beta_{1,1}, \beta_{1,0}, \beta_{0,0}\right) \\
& =\Psi\left(\alpha_{r, r}, \alpha_{r, r-1}, \ldots, \alpha_{r, 0}, \alpha_{r-1, r-1}, \alpha_{r-1, r-2}, \ldots, \alpha_{r-1,0}, \ldots, \alpha_{1,1}, \alpha_{1,0}, \alpha_{0,0}\right) \times M_{r}^{-1}
\end{aligned}
$$

where $M_{r}^{-1}$ is a lower triangular matrix with diagonal entries $\left\{(2 r+1)^{-1},(2 r)^{-1}, \ldots,(r+\right.$ $\left.1)^{-1} ;(2 r-1)^{-1},(2 r-2)^{-1}, \ldots, r^{-1} ; \ldots ; 7^{-1}, 6^{-1}, 5^{-1}, 4^{-1} ; 5^{-1}, 4^{-1}, 3^{-1} ; 3^{-1}, 2^{-1} ; 1^{-1}\right\}$. Then there exist some constants $m_{r^{\prime}, i^{\prime}}^{r, i} \in \mathbb{R}$ such that

$$
\beta_{r, i}=\Psi\left(\sum_{\left(r^{\prime}, i^{\prime}\right) \preceq(r, i)} m_{r^{\prime}, i^{\prime}}^{r, i} \alpha_{r^{\prime}, i^{\prime}}\right),
$$

and

$$
m_{r, 0}^{r, 0}=\frac{1}{r+1} .
$$

When $i>0$, the identity (6) implies

$$
\Psi\left(\alpha_{r, i}\right)-(r+i+1) \beta_{r, i}+i \beta_{r, i-1} \in \mathbb{R}_{r-1}[x, y] .
$$


By (13) and (15) we obtain

$$
\Psi\left(\alpha_{r, i}-(r+i+1) \sum_{\left(r^{\prime}, i^{\prime}\right) \preceq(r, i-1)} m_{r^{\prime}, i^{\prime}}^{r, i} \alpha_{r^{\prime}, i^{\prime}}+i \sum_{\left(r^{\prime}, i^{\prime}\right) \preceq(r, i-1)} m_{r^{\prime}, i^{\prime}}^{r, i-1} \alpha_{r^{\prime}, i^{\prime}}\right) \in \mathbb{R}_{r-1}[x, y],
$$

and therefore

$$
\alpha_{r, i}-(r+i+1) \sum_{\left(r^{\prime}, i^{\prime}\right) \preceq(r, i-1)} m_{r^{\prime}, i^{\prime}}^{r, i} \alpha_{r^{\prime}, i^{\prime}}+i \sum_{\left(r^{\prime}, i^{\prime}\right) \preceq(r, i-1)} m_{r^{\prime}, i^{\prime}}^{r, i-1} \alpha_{r^{\prime}, i^{\prime}} \in \mathbb{R}_{r-1}[x, y] .
$$

Then the coefficient of $\alpha_{r, 0}$ in (17) must be zero, i.e.,

$$
(r+i+1) m_{r, 0}^{r, i}=i m_{r, 0}^{r, i-1} .
$$

Finally by (14) and induction we obtain

$$
m_{r, 0}^{r, i}=\frac{r ! i !}{(r+i+1) !}
$$

for any $0 \leqslant i \leqslant r$. This means that, for a given $k$,

$$
\begin{aligned}
\left.\Psi^{-1}\left(x^{i}(x+2 y)^{j}\right)\right|_{x=k, y=2 n} & =\left.\Psi^{-1}\left(\beta_{i+j, i}\right)\right|_{x=k, y=2 n} \\
& =m_{i+j, 0}^{i+j, i}(k+2 n)^{i+j}+O\left(n^{i+j-1}\right) \\
& =\frac{i !(i+j) !(2 n)^{i+j}}{(2 i+j+1) !}+O\left(n^{i+j-1}\right)
\end{aligned}
$$

when $n \rightarrow \infty$. Then (4) holds by Theorem 3 .

On the other hand, when $i>0$, by comparing the coefficients of $\alpha_{r, i^{\prime}}\left(0 \leqslant i^{\prime} \leqslant i\right)$ in (17), we have

$$
1-(r+i+1) \sum_{i^{\prime}=0}^{i} m_{r, i^{\prime}}^{r, i}+i \sum_{i^{\prime}=0}^{i-1} m_{r, i^{\prime}}^{r, i-1}=0
$$

or equivalently,

$$
\sum_{i^{\prime}=0}^{i} m_{r, i^{\prime}}^{r, i}=\frac{1+i \sum_{i^{\prime}=0}^{i-1} m_{r, i^{\prime}}^{r, i-1}}{r+i+1}
$$

Therefore by (14) and induction we obtain

$$
\sum_{i^{\prime}=0}^{i} m_{r, i^{\prime}}^{r, i}=\frac{1}{r+1}
$$


for any $0 \leqslant i \leqslant r$. This means that, for a given $n$,

$$
\begin{aligned}
\left.\Psi^{-1}\left(x^{i}(x+2 y)^{j}\right)\right|_{x=k, y=2 n} & =\left.\Psi^{-1}\left(\beta_{i+j, i}\right)\right|_{x=k, y=2 n} \\
& =\sum_{i^{\prime}=0}^{i} m_{i+j, i^{\prime}}^{i+j, i} k^{i^{\prime}}(k+2 n)^{i+j-i^{\prime}}+O\left(k^{i+j-1}\right) \\
& =\frac{k^{i+j}}{(i+j+1)}+O\left(k^{i+j-1}\right)
\end{aligned}
$$

when $k \rightarrow \infty$. Then (5) holds by Theorem 3 .

\section{Remarks and discussions}

(1) The operator $\Psi$ defined in Section 2 and its inverse $\Psi^{-1}$ can be realized via a computer algorithm, as shown by the next program written for the computer algebra system Sage [25].

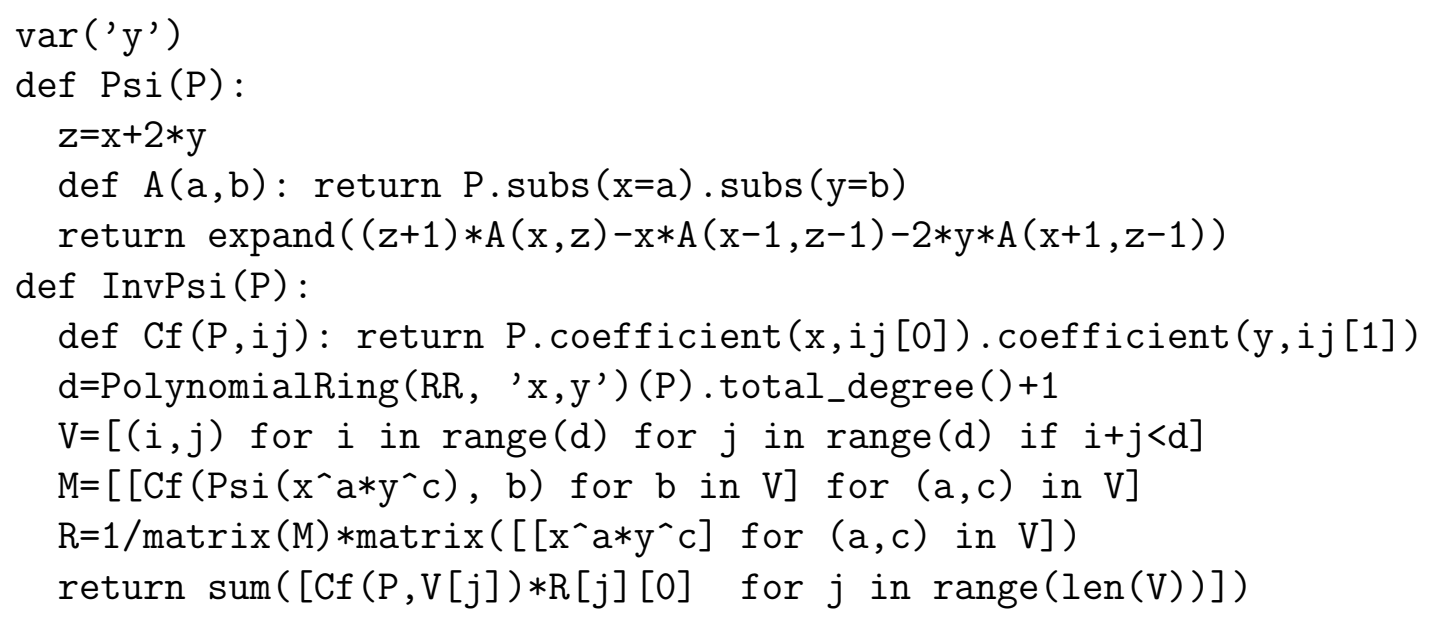

Here are two examples to verify the functions Psi and InvPsi:

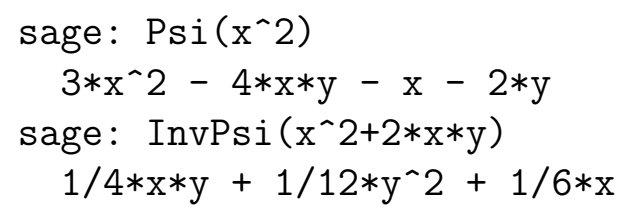

(2) As noted in Example 12, when $P(x, y)=\left(\begin{array}{l}y \\ r\end{array}\right)$, we have $\Psi^{-1}(P(x, x+2 y))=\frac{1}{r+1}\left(\begin{array}{l}y \\ r\end{array}\right)$. So what is $\Psi^{-1}(P(x, x+2 y))$ when $P(x, y)=\left(\begin{array}{l}x \\ r\end{array}\right)$ ? We cannot find a nice explicit formula for it. Instead we obtain

$$
\left.\Psi^{-1}\left(\left(\begin{array}{l}
x \\
r
\end{array}\right)\right)\right|_{x=0, y=2 n}=\frac{r !^{2} 2^{r}}{(2 r+1) !}\left(\begin{array}{l}
n \\
r
\end{array}\right)
$$


by comparing the identities (2) and (3). Is it possible to find a direct proof for (22) by the definition of $\Psi$ without Theorem 3 ?

(3) The technique developed in the present paper can be used for studying other problems on the averages of weight functions for oscillating tableaux. For each box $\square$ in the Young diagram of the partition $\lambda$, let $h_{\square}$ and $c_{\square}$ be its hook length and content respectively (see $[19,27]$ ). In a paper under preparation, by applying results from the study of difference operators on functions of partitions $[7,11,12,13]$, we will establish the following two explicit formulas with very complicated proofs for the average weights related to hook lengths and contents:

$$
\frac{2^{n} n !}{(2 n+1) !} \sum_{\left(\lambda^{0}, \ldots, \lambda^{l}\right) \in \mathcal{O} \mathcal{T}(\emptyset, 2 n)} \sum_{i=0}^{2 n} \sum_{\square \in \lambda^{i}} \prod_{1 \leqslant j \leqslant r}\left(h_{\square}^{2}-j^{2}\right)=\frac{(2 r) ! 2^{r}}{(2 r+3)(r+1) !}\left(\begin{array}{c}
n \\
r+1
\end{array}\right)
$$

and

$$
\frac{2^{n} n !}{(2 n+1) !} \sum_{\left(\lambda^{0}, \ldots, \lambda^{l}\right) \in \mathcal{O} \mathcal{T}(\emptyset, 2 n)} \sum_{i=0}^{2 n} \sum_{\square \in \lambda^{i}} \prod_{0 \leqslant j \leqslant r-1}\left(c_{\square}^{2}-j^{2}\right)=\frac{r ! 2^{r}}{(2 r+1)(2 r+3)}\left(\begin{array}{c}
n \\
r+1
\end{array}\right) .
$$

The above two identities can be seen as analogues of the Okada-Panova hook length formula $[22]$

$$
\frac{1}{n !} \sum_{|\lambda|=n} f_{\lambda}^{2} \sum_{\square \in \lambda} \prod_{1 \leqslant j \leqslant r}\left(h_{\square}^{2}-j^{2}\right)=\frac{(2 r) !(2 r+1) !}{r !(r+1) !^{2}}\left(\begin{array}{c}
n \\
r+1
\end{array}\right)
$$

and the Fujii-Kanno-Moriyama-Okada content formula [9]

$$
\frac{1}{n !} \sum_{|\lambda|=n} f_{\lambda}^{2} \sum_{\square \in \lambda} \prod_{0 \leqslant j \leqslant r-1}\left(c_{\square}^{2}-j^{2}\right)=\frac{(2 r) !}{(r+1) !}\left(\begin{array}{c}
n \\
r+1
\end{array}\right) .
$$

The above results suggest that there are several kinds of weight functions of oscillating tableaux whose averages have nice expressions such as polynomials. We want to summarize known results and find a theory for the averages of more general weight functions of oscillating tableaux in a paper under preparation.

(4) A skew oscillating tableau is a sequence of partitions $T=\left(\lambda^{0}, \lambda^{1}, \ldots, \lambda^{l}\right)$ such that $\left|\lambda^{i} / \lambda^{i+1}\right|=1$ or $\left|\lambda^{i+1} / \lambda^{i}\right|=1$ for each $0 \leqslant i \leqslant l-1$ without the condition that $\lambda^{0}=\emptyset$. The enumeration of skew oscillating tableaux was obtained by Roby [24]. So it is natural to ask the following question: Is there a generalization of Theorem 3 for skew oscillating tableaux? As pointed out in [14], the computation suggests that there are no simple formulas for skew oscillating tableaux.

(5) A strict partition is a partition whose parts are distinct to each other. We can define strict oscillating tableaux in a similar way. It will be interesting to find similar results for the enumerations and average weights for strict oscillating tableaux. 
(6) As explained in [14], oscillating tableaux are closely related to perfect matchings. The results on this topic can be found in $[2,5,6,15]$. It will be interesting to apply our results to perfect matchings in the future.

(7) The formula for the number of oscillating tableaux can be generalized to yield the number of walks of given length starting at the minimal element of a differential poset and ending at some fixed element (see [26]). Moreover, Hopkins and Zhang [14] use the theory of differential posets to obtain their result on oscillating tableaux. So it is natural to ask the following question: Do our results for Young's Lattice carry over to arbitrary differential posets? Although we don't use differential posets in this paper, we believe that our techniques should still work for general differential posets because they are inductive in nature. It would be interesting to extend the techniques in this paper to study general differential posets such as the Young-Fibonacci lattice (see [26]) in the future.

\section{Acknowledgements}

The authors really appreciate the valuable suggestions given by referees for improving the overall quality of the manuscript.

\section{References}

[1] J. Bandlow. An elementary proof of the hook formula. Electron. J. Combin., 15:\#R45, 2008.

[2] J. Bloom and S. Elizalde. Pattern avoidance in matchings and partitions. Electron. J. Combin., 20(2):\#P5, 2013.

[3] S. Burrill, J. Courtiel, E. Fusy, S. Melczer, and M. Mishna. Tableau sequences, open diagrams, and Baxter families. European J. Combin., 58: 144-165, 2016.

[4] S. Burrill. A generating tree approach to k-nonnesting arc diagrams. PhD thesis, Simon Fraser University, Burnaby, Canada, July 2014.

[5] W. Chen, E. Deng, R. Du, R. Stanley, and C. Yan. Crossings and nestings of matchings and partitions. Trans. Amer. Math. Soc., 359(4): 1555-1575, 2007.

[6] W. Chen and P. Guo. Oscillating rim hook tableaux and colored matchings. Adv. in Appl. Math., 48(2): 393-406, 2012.

[7] P.-O. Dehaye, G.-N. Han, and H. Xiong. Difference operators for partitions under the Littlewood decomposition. Ramanujan J., 44(1): 197-225, 2017.

[8] J. S. Frame, G. de B. Robinson, and R. M. Thrall. The hook graphs of the symmetric groups. Canadian J. Math., 6: 316-324, 1954.

[9] S. Fujii, H. Kanno, S. Moriyama, and S. Okada. Instanton calculus and chiral onepoint functions in supersymmetric gauge theories. Adv. Theor. Math. Phys., 12(6): 1401-1428, 2008.

[10] C. Greene, A. Nijenhuis, and H. S. Wilf. A probabilistic proof of a formula for the number of Young tableaux of a given shape. Adv. in Math., 31(1): 104-109, 1979.

[11] G.-N. Han and H. Xiong. Difference operators for partitions and some applications. Ann. Combin., 22(2): 317-346, 2018. 
[12] G.-N. Han and H. Xiong. New hook-content formulas for strict partitions. Algebraic Combin., 45(4) : 1001-1019, 2017.

[13] G.-N. Han and H. Xiong. Polynomiality of some hook-content summations for doubled distinct and self-conjugate partitions. arXiv:1601.04369, 2016.

[14] S. Hopkins and I. Zhang. A note on statistical averages for oscillating tableaux. Electron. J. Combin., 22(2):\#P2.48, 2015.

[15] A. Kasraoui and J. Zeng. Distribution of crossings, nestings and alignments of two edges in matchings and partitions. Electron. J. Combin., 13(1):\#R33, 2006.

[16] C. Krattenthaler. Bijective proofs of the hook formulas for the number of standard Young tableaux, ordinary and shifted. Electron. J. Combin., 2: \#R13, 1995.

[17] C. Krattenthaler. Oscillating tableaux and nonintersecting lattice paths. J. Statist. Plann. Inference, 54(1): 75-85, 1996.

[18] C. Krattenthaler. Bijections between oscillating tableaux and (semi)standard tableaux via growth diagrams. J. Combin. Theory Ser. A, 144: 277-291, 2016.

[19] I. G. Macdonald. Symmetric Functions and Hall Polynomials. Oxford Mathematical Monographs, The Clarendon Press, Oxford University Press, New York, second edition, 1995.

[20] J.-C. Novelli, I. Pak, and A. V. Stoyanovskii. A direct bijective proof of the hooklength formula. Discrete Math. Theor. Comput. Sci., 1(1): 53-67, 1997.

[21] S. Okada. Pieri rules for classical groups and equinumeration between generalized oscillating tableaux and semistandard tableaux. Electron. J. Combin., 23(4):\#P4.43, 2016.

[22] G. Panova. Polynomiality of some hook-length statistics. Ramanujan J., 27(3): 349-356, 2012.

[23] I. Pak and P. Alexander. Oscillating tableaux, Sp $\times$ Sq-modules, and RobinsonSchensted-Knuth correspondence. Proceedings of FPSAC 96, 1998.

[24] T. Roby. Applications and extensions of Fomin's generalization of the RobinsonSchensted correspondence to differential posets. PhD thesis, Massachusetts Institute of Technology, September 1991.

[25] The Sage-Combinat community. Sage-Combinat: enhancing Sage as a toolbox for computer exploration in algebraic combinatorics. 2008, http://combinat. sagemath. org.

[26] R. Stanley. Differential posets. J. Amer. Math. Soc., 1(4): 919-961, 1988.

[27] R. Stanley. Enumerative Combinatorics, Volume 2. Cambridge University Press, 2001.

[28] R. Stanley. Algebraic Combinatorics: Walks, Trees, Tableaux, and More. Springer, New York, 2013.

[29] S. Sundaram. On the combinatorics of representations of $S p(2 n, \mathbb{C}) . \mathrm{PhD}$ thesis, Massachusetts Institute of Technology, April 1986. 